\title{
Ablation of Distal Ulna as an Index Procedure in A Complex Open Distal Radius-Ulna Fracture with Defective Soft Tissue Coverage
}

\author{
Rudy Reza Johan*, Tan Jin Aun and Juzaily Fekry Leong \\ Department of Orthopaedic and Tramatology, Malaysia
}

Received: 此 December 21, 2019; Published: 制 January 28, 2019

*Corresponding author: Rudy Reza Johan, Department of Orthopaedic and Tramatology, Hospital Sultanah Aminah Johor Bahru, Malaysia

\begin{abstract}
Unstable open fracture of the distal ulna with severe soft tissue disruption in association with distal end radius fracture may be beyond the scope of fixation. There is little scientific support to guide the management of this devastating fracture. We opted to perform a distal ulna resection in a young patient at the time of definitive rigid fixation of the distal radius and successfully managed to close the wound primarily. This technique has provided a reproducibly satisfactory reconstruction and maintenance of reasonably near normal wrist joint biomechanics.
\end{abstract}

Keywords: Comminuted Open Distal Ulna Fracture; Soft Tissue Defect; Darrach Resection

Abbreviations: ECU: Extensor Carpi Ulnaris; EDQP: Extensor Digiti Quinti Proprius; TFCC: Triangular Fibrocartilage Complex

\section{Introduction}

Open comminuted intra-articular fractures of the distal ulna with significant skin defect associated with fractures of the distal radius is rare. This prevailing fracture with concomitant destabilizing injury to the primary and secondary radio-ulnar ligamentous structures is invariably associated with distal radioulna joint instability. Anatomic fragment reduction and stable retention of the distal ulna may not be feasible by osteosynthesis. Extensive zone of injury at the wrist and forearm oppresses durable and viable soft tissue coverage. There is minimal guidance in the current literature regarding management of this difficult fractures. This case report describes the pivotal role of distal ulna resection in irreparable unstable fractures with soft tissue inadequacy.

\section{Case Report}

A 33-year-old male nurse who was involved in a road traffic accident had sustained a distal end left radius fracture AO 2.3-C1; and open comminuted fracture of the distal left ulna Biyani classification Type IV [1] with distal radio-ulnar joint instability as well as a contaminated wound measuring $2.5 \times 1.5 \mathrm{~cm}$. The exposed ulna head was devoid of periosteum. The right hand dominant motorcyclist also acquired a radial head fracture Mason type 1 and fracture base of 5 th metacarpal bone of the ipsilateral left upper extremity.
An urgent thorough osseous and soft tissue debridement was performed under general anesthesia in the emergency theatre. The distal radius was approached via the modified Henry's, reduced and temporarily fixed with 2 cross k-wires and augmented with a spanning external fixator across the wrist. The distal ulna was approached dorsally incorporating the traumatic wound. Dorsal cutaneous branch of the ulna nerve was identified and preserved (Figure 1). The extensor retinaculum and distal radio-ulnar joint capsule was gravely damaged.

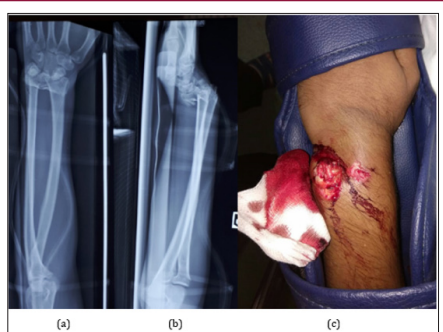

Figure 1: (a;b) Radiographs depicting complex displaced fracture left distal radius and ulna, fracture of the left radial head and base of left $5^{\text {th }}$ metacarpal bone.

(c) Traumatic wound at the dorsum of the left wrist revealing the distal ulna. 
The comminuted fragments of the distal ulna were reduced and temporarily fixed with multiple intrafocal pinning. Reduction of the ulna head into the sigmoid notch was laborious and recalcitrantly dislocated dorsally. The size of the wound after debridement had increased to $3.0 \times 4.5 \mathrm{~cm}$ exposing the underlying extensor carpi ulnaris (ECU) and extensor digiti quinti proprius (EDQP), their sheaths and the distal ulna. Closed reduction and percutaneous pinning of the base of the 5th metacarpal was done. The radial head was managed conservatively. The forearm was immobilized in a sugar thong slab. Intravenous cefuroxime and gentamycin were administered. Despite regular hydrogel dressing of the wound, the exposed ulna was dry and mildly necrotic. Definitive stable fixation of the radius via a variable angle locking plate was subsequently performed. The distal ulna was approached from the previous wound dorsally.

The multi- fragmented distal ulna was osteotomised using a power oscillating saw $1 \mathrm{~mm}$ proximal to the fracture approximating $1.0 \mathrm{~cm}$ proximal to the sigmoid notch and then bevelled in a geometric angle distally and ulnarly. The distal pole, seat and entire styloid were sacrificed. Complete decompression of the ulnocarpal and distal radio-ulna joints was executed. There was no adequate periosteal sleeve to be elevated and closed diligently. The triangular fibrocartilage complex (TFCC) was detached from the basi-styloid. The damaged transverse radio-ulnar branch of the ulnar nerve was transected and cauterised. Dorsal capsular reinforcement was performed by utilising the remaining retinacular flap. Generally, the retinaculum was not amenable to meticulous reconstruction due to the extensive soft tissue trauma. Fortunately, part of the remaining 5th compartment was imperatively anchored to the radial side of the 4th compartment. The EDQP and ECU were transposed dorsal to the extensor retinaculum.

Soft tissue reconstruction to counteract winging of the ulna stump advocating the ECU tendon could not be enforced. The skin was successfully closed routinely eliminating the defect. The extremity was maintained on a long splint age till 8 weeks. Oedema control and full digital motion exercise was commenced early to prevent stiffness. The wound healed well. Regardless of the radical element of this procedure, this patient endured restoration of a pain- free forearm rotation. The prono-supination arc was $60-90^{\circ}$. His wrist flexion was $50^{\circ}$ and extension was $20^{\circ}$. The digital motion of all the small joints of the fingers and thumb were full. He can conduct all the basic function of the hands (Figure 2). However as expected, he has some weakness. His lateral pinch was $5.13 \mathrm{~kg} / \mathrm{F}$ as compared to the right side of $7.26 \mathrm{~kg} / \mathrm{F}$. His grip strength measured by Jamar dynamometer was noted to be diminished with the left side recording $9.3 \mathrm{~kg} / \mathrm{F}$ as compared to the normal right side at $22.7 \mathrm{~kg} / \mathrm{F}$. There was no numbness or paranesthesia. After 6 months of follow up, patient did not develop inherent complications comprising of distal ulna stump instability, impingement of the stump against the medial radial cortex, ulnocarpal translocation, tendon ruptures and mechanical locking. No additional surgical procedure was warranted (Figure 3).

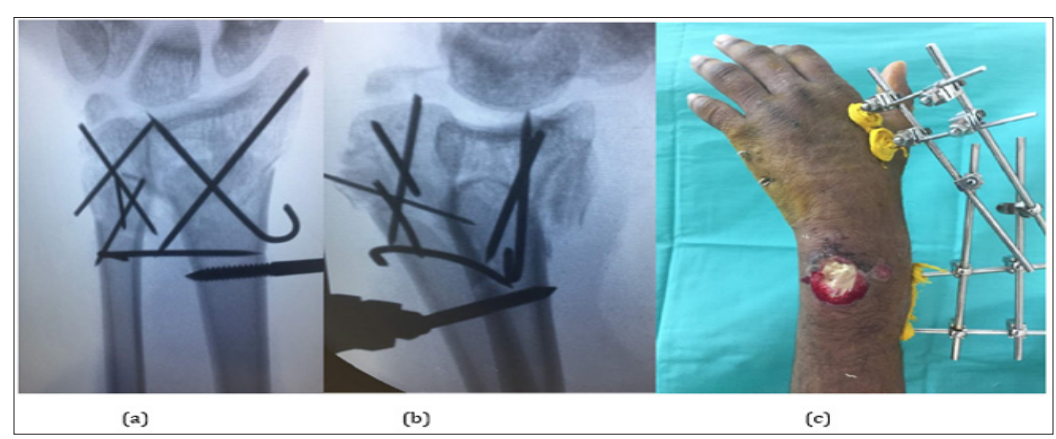

Figure 2: $(a ; b)$ Multiple intra-focal k-wiring of the shattered distal ulna with dorsal dislocation. (c) Skin loss exposing the ECU sheath and distal ulna.

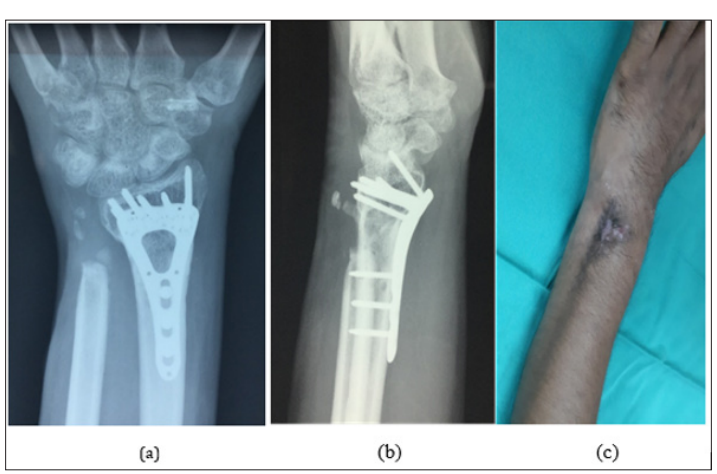

Figure 2: $(a ; b)$ The distal radius achieved osseous union. There was no radiographic evidence of ulnar stump instability or ulnar carpal translocation.

(c) Healed wound with mild hyperpigmentation over the dorso-ulna aspect of the left wrist. 


\section{Discussion}

Good to excellent results have been reported in younger patients with increased demands. [2] This technique yields favourable outcomes when compared with internal locked fixation which may require re-operation for removal of symptomatic ulnarsided hardware and revision fixation with bone grafting for a distal ulna non-union [3]. Loss of reduction parameters after attempted poor fixation can contribute to ulnocarpal abutment syndrome and DRUJ arthrosis with persistent ulna wrist pain [4]. Inability to reconstruct a healthy flap to cover the bone- implant construct may lead to potential catastrophic flap failure and debilitating infection.

This patient relishes a satisfactory functional outcome in his activities of daily living using his left hand which was feared to be dysfunctional. Weakness should not be essentially regarded as a complication. In a reported study no patients found their activities disturbed by reduced grip strength. Long term follow-up of almost 2 years in this series showed gradual improvement in the grip power [5]. With the accurate indication and diligent surgical workmanship, the classical Darrach distal ulna resection can be extended as an indispensable procedure when there is failure to achieve anatomic restoration of the ulna head to sigmoid notch articular relationship, compounded with unhealthy ulna bone and soft tissue scarcity.

\section{References}

1. Biyani A, Simison AJ, Klenerman L (1995) Fractures of the distal radius and ulna. J Hand Surg Br 20(3): 357-364.

2. Seitz WH, Raikin SM (2007) Resection of comminuted ulna head fragments with soft tissue reconstruction when associated with distal radius fractures. Tech Hand Up Extrem Surg 11(4): 224-230.

3. Dennison DG (2007) Open reduction and internal locked fixation of unstable distal ulna fractures with concomitant distal radius fracture. J Hand Surg Am 32(6): 801-805.

4. Knirk JL, Jupiter JB (1986) Intra-articular fractures of the distal end of the radius in young adults. J Bone Joint Surg Am 68(5): 647-659.

5. H.Yoneda , K Watanabe (2014) Primary excision of the ulnar head for fractures of the distal ulna associated with fractures of the distal radius in severe osteoporotic patients. J Hand Surgery Europe 39: 293-299.

\section{ISSN: 2574-1241}

DOI: 10.26717/BJSTR.2019.13.002439

Rudy Reza Johan. Biomed J Sci \& Tech Res

(C) (P) This work is licensed under Creative

Submission Link: https://biomedres.us/submit-manuscript.php

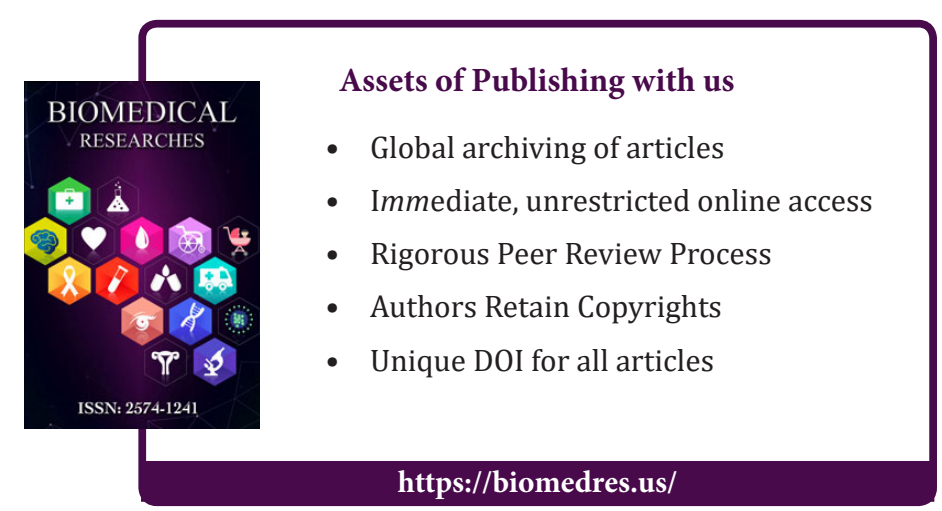

